\title{
Interaction of CL-20 and Zinc - A DFT Treatment
}

\section{Lemi Türker}

Department of Chemistry, Middle East Technical University, Üniversiteler, Eskişehir Yolu No: 1, 06800 Çankaya/Ankara, Turkey; e-mail: lturker@gmail.com; lturker@metu.edu.tr

\begin{abstract}
CL-20 or HNIW is a novel, caged-type explosive material having six nitramine groups. In the present study, interaction of CL-20 with zinc atom has been investigated within the constraints of density functional theory at the level of B3LYP/6-31+G(d,p). Zinc, in chemistry is known as a good reducing metal for many functional groups, presently not only reduces one of the nitro groups but also causes cleavage of one of the nitramine bond (from the base ring) and the $\mathrm{C}-\mathrm{N}$ bond of the cage. The zinc atom acquires some positive charge after all.
\end{abstract}

\section{Introduction}

2,4,6,8,10,12-Hexanitro-2,4,6,8,10,12-hexaazaisowurtizane which is a cage-like compound, (commonly called CL-20 or HNIW) was synthesized in 1987 by Nelsen [13]. CL-20 is a novel, high-density $\left(2.04 \mathrm{~g} / \mathrm{cm}^{3}\right)$ cyclic nitramine (Figure 1) which can be used as an energetic component in explosive formulations and propellants. Its standard molar enthalpy of formation is of the order of about $419 \mathrm{~kJ} / \mathrm{mol}$ [3-5]. It has several polymorphs [4]. The epsilon-form is the one having the highest density and greatest thermal stability among its polymorphs $[5,6]$. Its gas phase dissociation yields high concentration of $\mathrm{NO}_{2}$ and a few ring fragments with nitro groups (the ring fragments of $[\mathrm{CxHyNz}]^{+}$have been detected [7, 8]. Cocrystal formation of CL-20 with HMX has been reported [9]. Some polymer based explosive (PBX) formulations having CL-20 have

\section{Received: July 10, 2019; Accepted: August 8, 2019}

Keywords and phrases: CL-20, HNIW, zinc, reduction, DFT.

Copyright (C) 2019 Lemi Türker. This is an open access article distributed under the Creative Commons Attribution License, which permits unrestricted use, distribution, and reproduction in any medium, provided the original work is properly cited. 

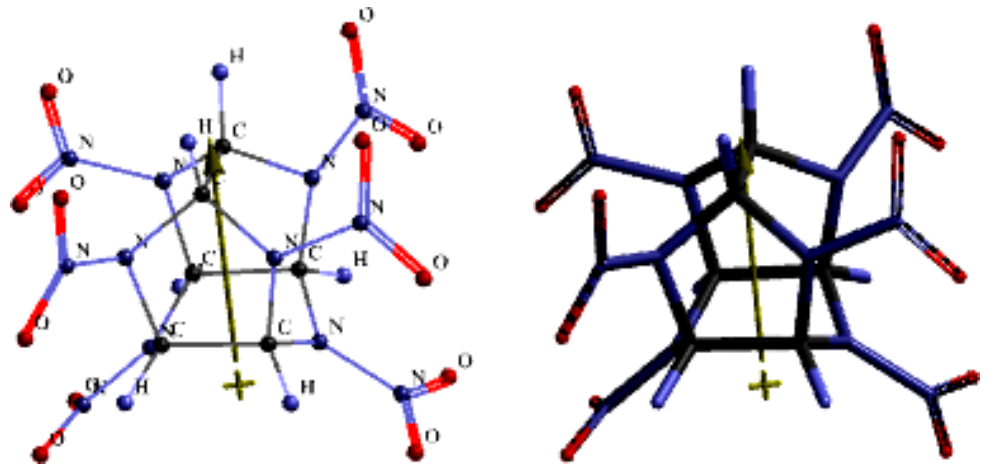

Figure 1. Structure of CL-20.

been established [10]. Thermally induced damage in CL-20 was reported by Tian et al. $[11,12]$ and also by Zeman and coworkers [13]. Its shock sensitivity in a polyisobutylene matrix was investigated [14, 15]. Temperature-dependent shock initiation of CL-20based high explosives was investigated by Pi et al. [16]. A detailed quantum chemical analysis for the instability of CL-20 exposed to structural variations was reported [17].

Aluminum is widely used in explosive compositions for high performance and high blast including CL-20 [18]. Magnesium is an important ingredient of high-energy mixtures containing an oxidant and a reductant. An oxidant contained in mixture facilitates its combustion without access to air oxygen, which means that the desired special effect is possible. Magnesium containing compositions have found a broad range of applications in show and military pyrotechnics, in rocket propellants and explosive mixtures [19].

Composites of porous metal (nickel, aluminum and magnesium) and nano-scale aluminum powder and CL-20 have been synthesized with UV (ultraviolet) ink method, respectively [20] and the structure, morphology, infrared characteristic, thermal decomposition of composites were characterized. Chan and Turner patented various thermobaric explosive formulations with metastable mechanical alloys which include nano-sized $\mathrm{Al}-\mathrm{Mg}, \mathrm{Al}-\mathrm{Mg}-\mathrm{H}, \mathrm{B}-\mathrm{Mg}, \mathrm{Al}-\mathrm{B}, \mathrm{Ti}-\mathrm{B}$, incorporate high energy explosive material including, hexanitrohexaaza-isowurtzitane (CL-20) [21].

In the present study, the interaction of $\mathrm{Zn}$ atom and CL-20 explosive is considered quantum chemically within the limitations of density functional theory (DFT). 


\section{Method of Calculation}

The initial geometry optimizations of all the structures leading to energy minima were achieved by using MM2 (molecular mechanics) method followed by semi-empirical PM3 self-consistent field molecular orbital (SCF-MO) method [22, 23] at the restricted level [24]. Then, the geometry optimizations were achieved by using various restricted Hartree-Fock (RHF) methods successively and finally optimizing within the framework of density functional theory (DFT, B3LYP) $[25,26]$ at the level of $6-31+G(d)$. The exchange term of B3LYP consists of hybrid Hartree-Fock and local spin density (LSD) exchange functions with Becke's gradient correlation to LSD exchange [27, 28]. The correlation term of B3LYP consists of Vosko, Wilk, Nusair (VWN3) local correlation functional [29] and Lee, Yang, Parr (LYP) correlation correction functional [29].

Vibrational analyses and the calculation of total electronic energies were performed using the same type of calculations (B3LYP/6-31+G(d)). The normal mode analysis yielded no imaginary frequencies, which indicates that each compound had at least a local minimum on the potential energy surface. The total electronic energies were obtained by single point (B3LYP/6-31+G(d)) calculations over DFT optimized geometries and corrected for zero point vibrational energies (ZPE). All the computations were performed using Spartan 06 software [30].

\section{Results and Discussion}

Zinc atom has $1 s^{2} 2 s^{2} 2 p^{6} 3 s^{2} 3 p^{6} 3 d^{10} 4 s^{2}$ electronic configuration in its ground state. It is a closed shell system and donating its $4 \mathrm{~s}$ electrons it may reduce certain organic functional groups. Zinc atom also forms some organozinc compounds, most of them exhibit spontaneous ignition in air and makes them difficult to handle [31]. The ionization potential of zinc is $9.36 \mathrm{eV}$ and $17.89 \mathrm{eV}$, respectively for the first and second oxidation states [32].

Figure 2 shows the optimized structures of CL-20+Zn composite. As seen there, the zinc atom not only causes the rupture of $\mathrm{N}-\mathrm{NO}_{2}$ bond $\left(\mathrm{N}-\mathrm{NO}_{2}\right.$ distance is $4.20 \AA$ ) but also cleaves one of the $\mathrm{C}-\mathrm{N}$ bonds of the cage. Note that the $\mathrm{NO}_{2}$ moiety expelled originally linked to the hexagonal base ring (piperazine ring) of CL-20. Figure 3 shows the bond lengths /distances of the composite system. One of the $\mathrm{C}-\mathrm{N}$ bonds belonging to 
one of the pentagonal rings is elongated $(3.60 \AA)$. Note that the carbon end of that bond originates from the hexagonal base ring (piperazine ring).
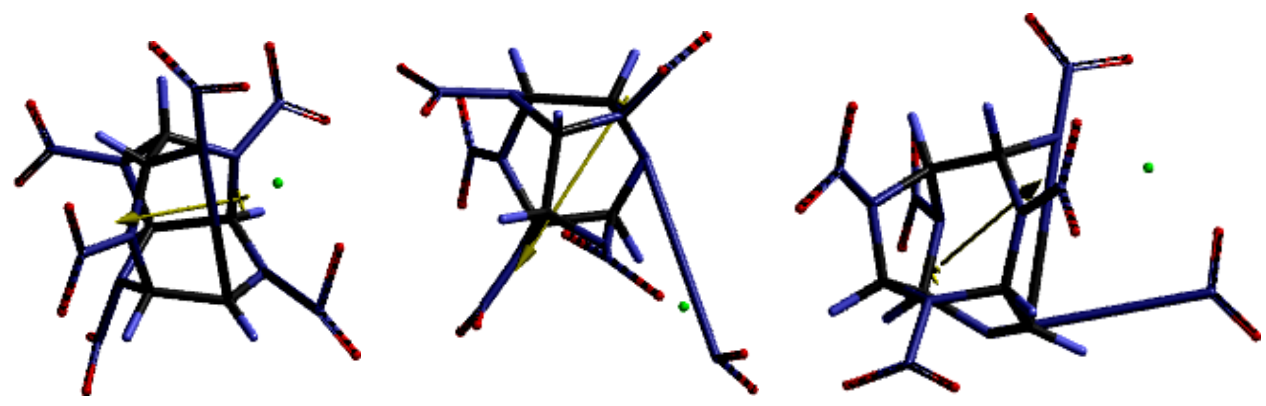

Figure 2. Optimized structure of $\mathrm{Cl}-20+\mathrm{Zn}$ composite (from different angles of view).

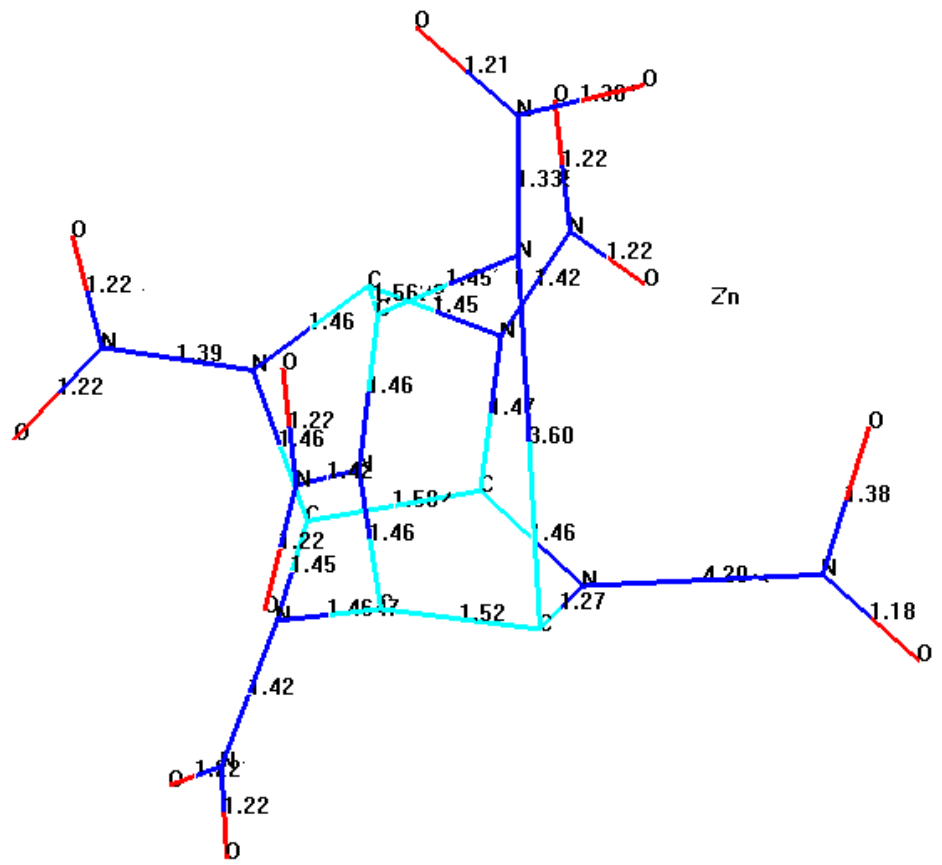

Figure 3. Calculated bond lengths/distances $(\AA)$ of the composite (hydrogens omitted).

Figure 4 shows the ESP (electrostatic potential) and the natural charges on the atoms of CL-20 and CL-20+Zn composite system. Note that the ESP charges are obtained by the program based on a numerical method that generates charges that reproduce the electrostatic potential field from the entire wavefunction [30]. Also note that the expelled 
$\mathrm{NO}_{2}$ group has negative overall charge. The nitrogen atom on the other side of the cleaved nitramine bond also has some negative charge, whereas the zinc atom acquires some positive charge.
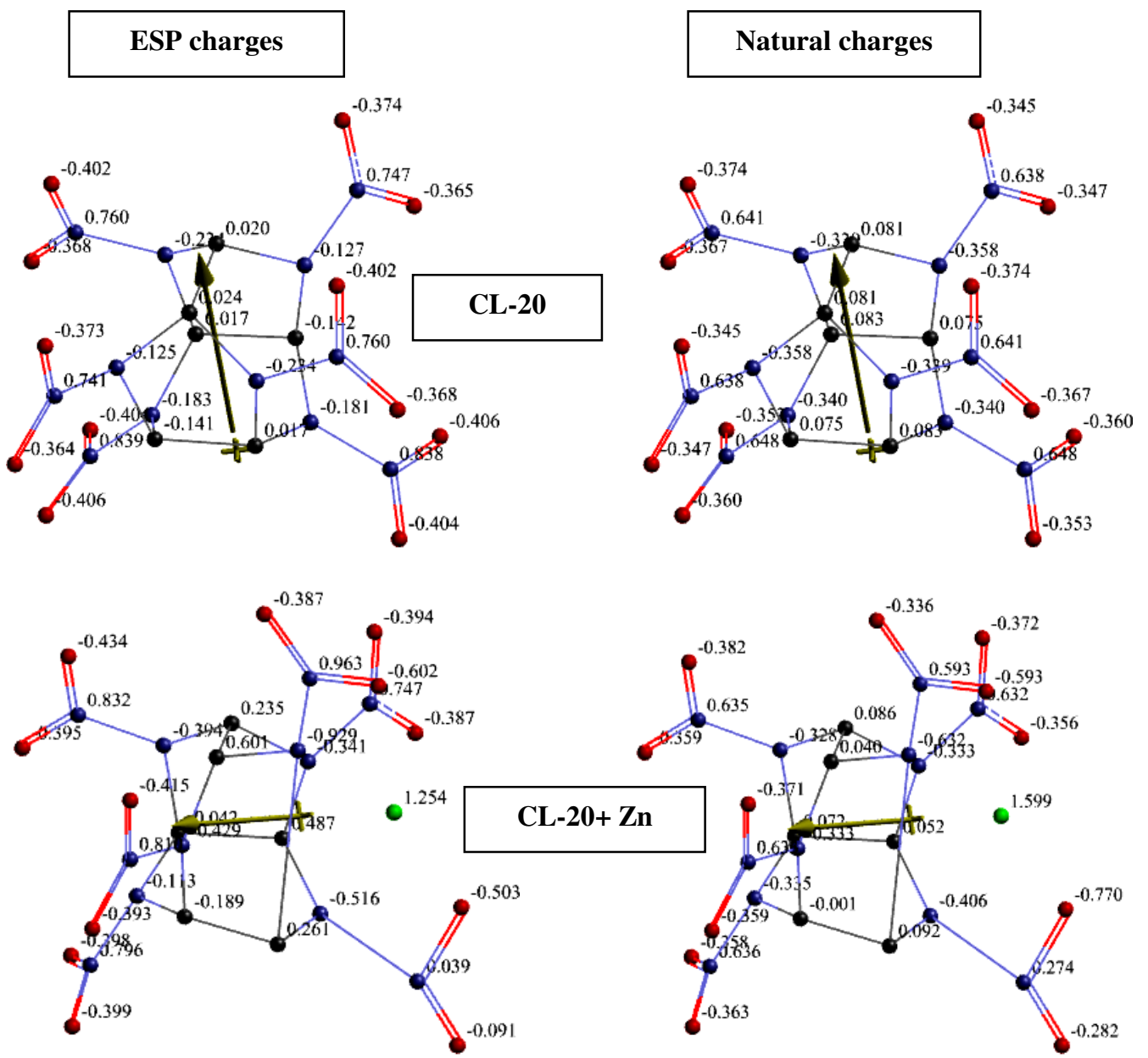

Figure 4. ESP and natural charges on the atoms of the systems considered (hydrogens omitted).

Figure 5 shows the IR spectrums of CL-20 and the composite. The C-H stretchings occur about $3172 \mathrm{~cm}^{-1}$. The peaks around $1662 \mathrm{~cm}^{-1}$ stand for the asymmetric N-O stretching of the nitro groups. The $\mathrm{C}-\mathrm{H}$ bendings are at $1304 \mathrm{~cm}^{-1}$. 


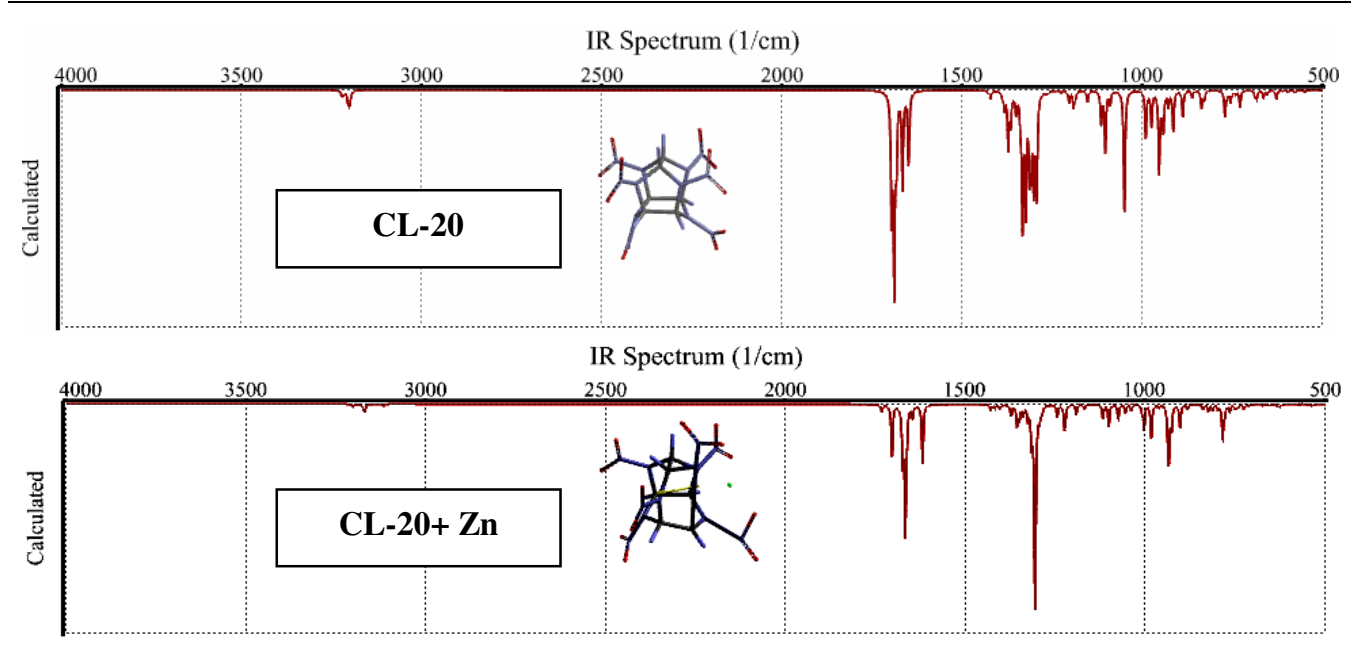

Figure 5. IR spectrums of CL-20 and CL-20+Zn composite.

Figure 6 depicts some of the molecular orbital energy levels of the systems of concern. As seen there the composite has appreciably high HOMO energy level as compared to CL-20 (see Table 1).
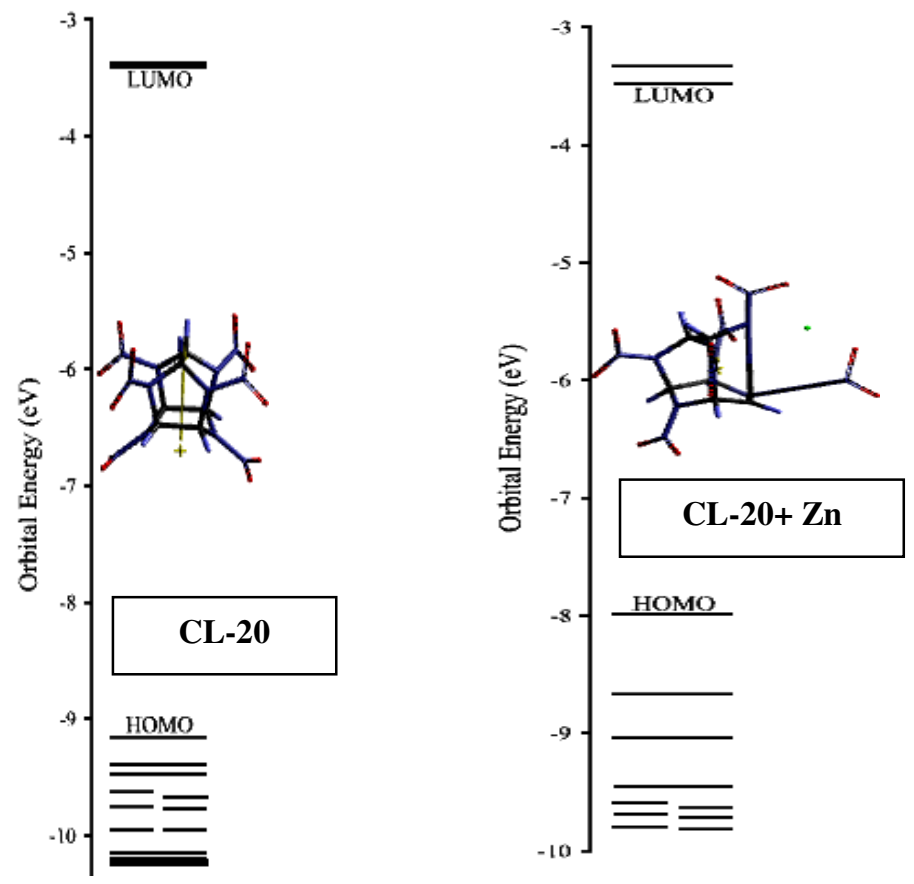

Figure 6. Some molecular orbitals of CL-20 and the composite. 
Table 1. The frontier molecular orbital energies and interfrontier molecular orbital energy gaps $(\Delta \varepsilon)$.

\begin{tabular}{|l|c|c|c|}
\hline & HOMO & LUMO & $\Delta \boldsymbol{\varepsilon}$ \\
\hline CL-20 & -883.33 & -332.59 & 550.74 \\
\hline CL-20+Zn & -770.95 & -335.83 & 435.12 \\
\hline Energies in kJ/mol.
\end{tabular}

Figure 7 shows the patterns of frontier molecular orbitals (HOMO and LUMO) and the NEXTHOMO and NEXTLUMO (HOMO-1 and LUMO+1). As seen in the figure, the HOMO of the composite is supplied by the departed $\mathrm{NO}_{2}$ moiety. All the other orbitals shown in the figure have contributions originating from the cage atoms and the departed $\mathrm{NO}_{2}$ group supplies either very small contribution or nil.
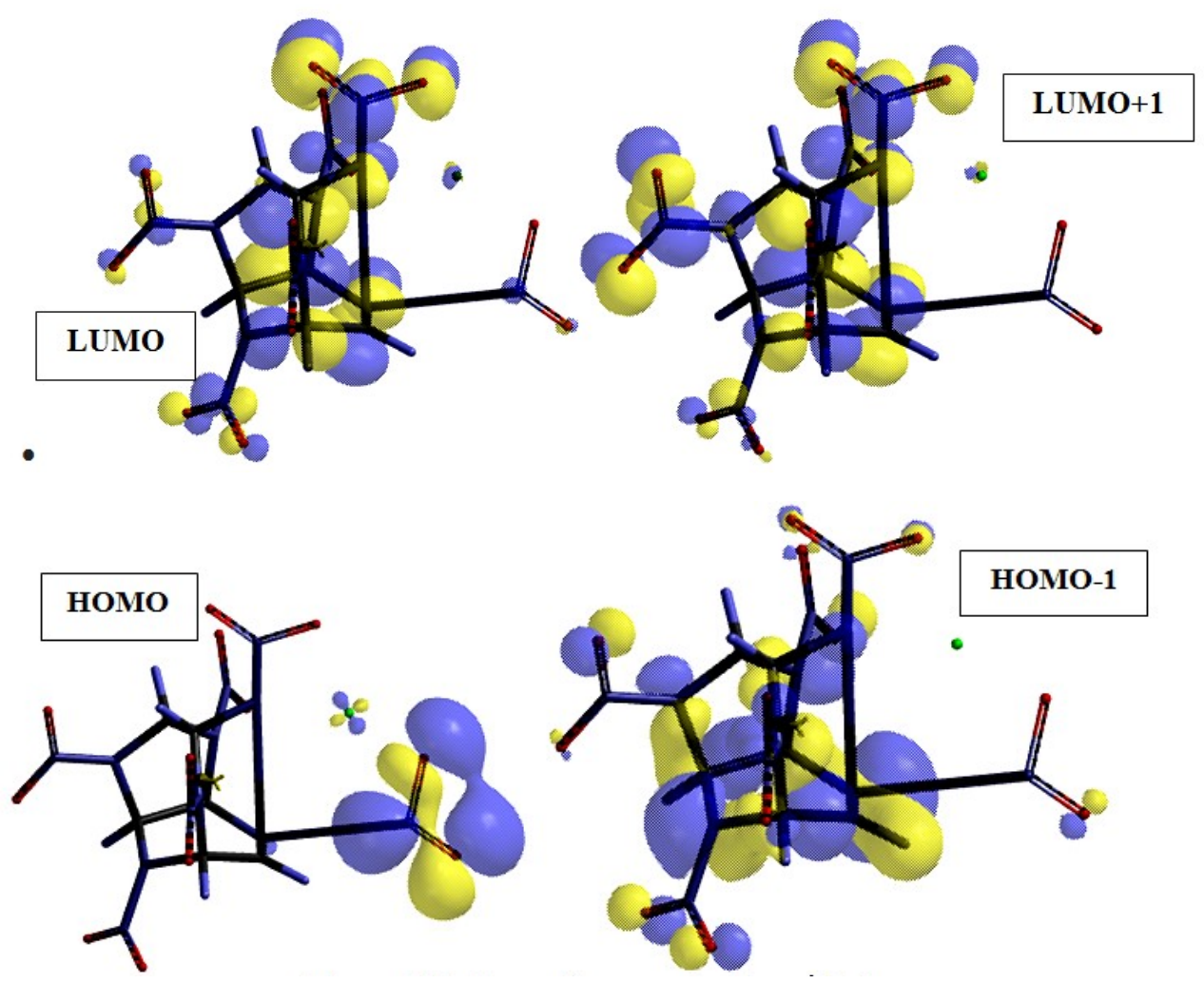

Figure 7. Patterns of some molecular orbitals. 
Figure 8 stands for the electrostatic potential maps of CL-20 and the composite. In the figure the blue and red/orange regions stand for positive and negative potential fields, respectively. Thus, in the case of composite the expelled $\mathrm{NO}_{2}$ is associated with some negative field.
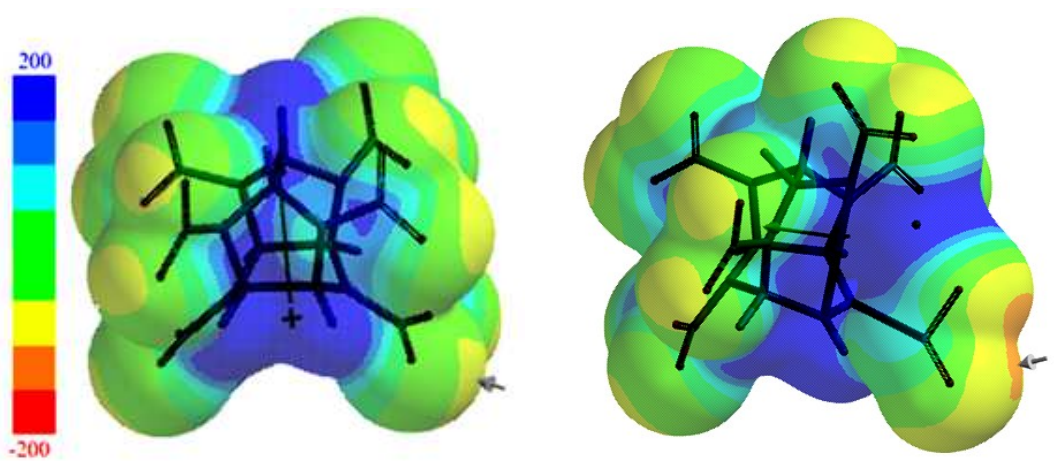

Figure 8. Electrostatic potential maps of CL-20 and the composite.

Figure 9 shows the local ionization maps of CL-20 and the composite. Note that the local ionization potential provides a measure of the relative ease of electron removal (ionization) at any location around a molecule. A map of local ionization potential onto an electron density surface reveals those regions from which electrons are most easily ionized. Local ionization potential maps reveal sites that are susceptible to electrophilic attack [33]. Thus in the figure, the dark and light blue regions stand for the least and moderately susceptible electrophilic attack.
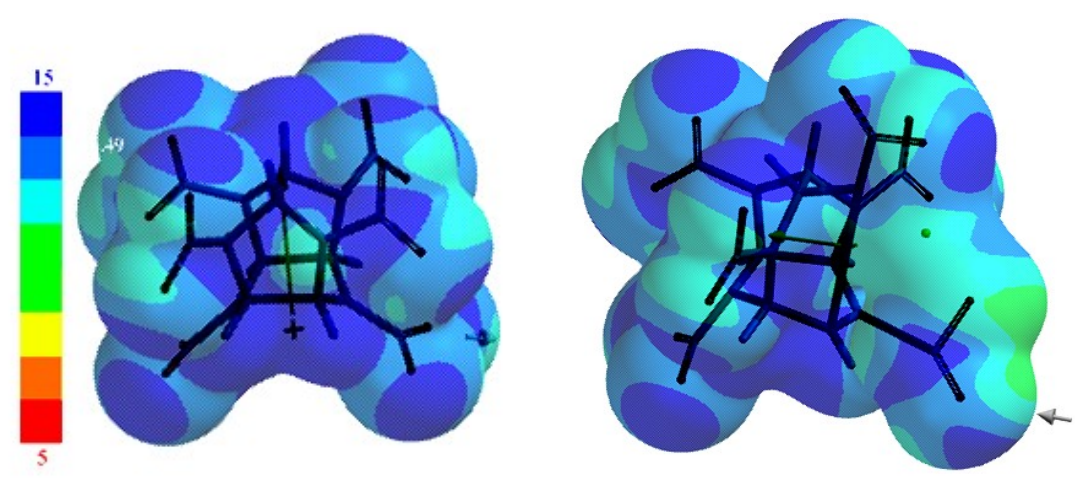

Figure 9. Local ionization potential maps of CL-20 and the composite. 


\section{Conclusion}

Within the restrictions of the density functional theory at the applied level the present study indicates that transfer of some electron population from the zinc atom to highly metastable CL-20 cage occurs and causes some bond cleavages. One of the nitramine $\mathrm{N}-\mathrm{NO}_{2}$ bonds of the base ring as well as the $\mathrm{C}-\mathrm{N}$ bond of the pentagonal ring (where the carbon atom of the broken bond was initially common to the base and the pentagonal rings) are broken. The study indicates that zinc element should not be employed together with CL-20 for the purpose of obtaining increased heat content of a composite system.

\section{References}

[1] A. T. Nielsen, Caged polynitramine compound, US Patent 5693794, 1997.

[2] A. T. Nielsen, A. P. Chafin, S. L. Christian, D. W. Moore, M. P. Nadler, R. A. Nissan, D. J. Vanderah, R. D. Gilardi, C. F. George and J. L. Flippen-Anderson, Synthesis of polyazapolycyclic caged polynitramines, Tetrahedron 54 (1998), 11793-11812. https://doi.org/10.1016/S0040-4020(98)00711-X

[3] Y. Bayat, J. Mokhtari, N. Farhadian and M. Bayat, Heteropolyacids: An efficient catalyst for synthesis of CL-20, Journal of Energetic Materials 30(2) (2012), 124-134. https://doi.org/10.1080/07370652.2010.549539

[4] P. Politzer and J. S. Murray, Energetic Materials, Part 1, Decomposition, Crystal and Molecular Properties, Amsterdam: Elsevier, 2003.

[5] M. Foltz, C. L. Coon, F. Garcia and A. L. Nichols, III, The thermal stability of the polymorphs of hexanitrohexaazaisowurtzitane, Part I, Propell. Explos. Pyrotech. 19 (1994), 133-145.

[6] X. Jiang, X. Guo, H. Ren and Q. Jiao, Preparation and characterization of desensitized $\varepsilon$-HNIW in solvent-antisolvent recrystallizations, Cent. Eur. J. Energ. Mater. 9 (2012), 219-236.

[7] R. J. Doyle, Jr., The gas-phase dissociation of a new polyazapolycyclic nitramine: Hexanitrohexaazaisowurtzitane, Org. Mass Spectrom. 26 (1991), 723-726. https://doi.org/10.1002/oms.1210260812

[8] D. G. Patil and T. B. Brill, Thermal decomposition of energetic materials 53. Kinetics and mechanism of thermolysis of hexanitroazaisowurtizitane, Combust. Flame 87 (1991), 145-151. https://doi.org/10.1016/0010-2180(91)90164-7 
[9] O. Bolton, L. R. Simke, P. F. Pagoria and A. J. Matzger, High power explosive with good sensitivity: A 2:1 cocrystal of CL-20:HMX, Cryst. Growth Des. 12 (2012), 4311-4314. https://doi.org/10.1021/cg3010882

[10] U. R. Nair, S. N. Asthana, A. S. Rao and B. R. Gandhe, Advances in high energy materials, Defence Science Journal 60(2) (2010), 137-151. https://doi.org/10.14429/dsj.60.327

[11] Q. Tian, G. Yan, G. Sun, C. Huang, L. Xie, B. Chen, M. Huang, H. Li, Y. Liu and J. Wang, Thermally induced damage in hexanitrohexaazaisowurtzitane, Cent. Eur. J. Energ. Mater. 10 (2013), 359-369.

[12] D. Wang, B. Gao, G. Yang, F. Nie and H. Huang, Preparation of CL-20 explosive nanoparticles and their thermal decomposition property, Journal of Nanomaterials 2016 (2016), Article ID 5462097, 7 pp. https://doi.org/10.1155/2016/5462097

[13] S. Zeman, Q-L. Yan and M. Vlacek, Recent advances in the study of the initiation of energetic materials using characteristics of their thermal decomposition. Part I. Cyclic nitramines, Cent. Eur. J. Energ. Mater. 11 (2014), 173-189.

[14] V. Pelikan, S. Zeman, Q-L. Yan, M. Erben, A. Elbeih and Z. Akstein, Concerning the shock sensitivity of cyclic nitramines incorporated into a polyisobutylene matrix, Cent. Eur. J. Energ. Mater. 11 (2014), 219- 235.

[15] R. W. Molt, Jr., R. J. Bartlett, T. Watson, Jr. and A. P. Bazanté, Conformers of CL-20 explosive and ab initio refinement using perturbation theory: implications to detonation mechanisms, J. Phys. Chem. A 116(49) (2012), 12129-12135.

https://doi.org/10.1021/jp305443h

[16] Z. Pi, L. Chen and J. Wu, Temperature-dependent shock initiation of CL-20-based high explosives, Cent. Eur. J. Energ. Mater. 14(2) (2017), 361-374. https://doi.org/10.22211/cejem/68392

[17] L. Türker, Instability of CL-20 exposed to the effects of $\alpha$-particle, Indian Journal of Chem. A 54 (2015), 858-866.

[18] S. M. Nicolich, C. Capellos, W. A. Balas, J. D. Akestar and R. L. Hatch, High-blast explosive compositions containing particulate metal, US Patent 8168016, 2012.

[19] L. Türker, Thermobaric and enhanced blast explosives (TBX and EBX), Defence Technology 12(6) (2016), 423-445. https://doi.org/10.1016/j.dt.2016.09.002

[20] Z. H. Li, H. Ren, Q. J. Jiao and J. Dong, Fabrication and characterization in composites of nanoenergetic materials and porous metal with UV ink method, Integrated Ferroelectrics 152 (2014), 73-80. https://doi.org/10.1080/10584587.2014.901855 
[21] M. L. Chan and A. D. Turner, High energy blast explosives for confined spaces, US Patent 20070113939, 2007.

[22] J. J. P. Stewart, Optimization of parameters for semiempirical methods I. Method, $J$. Comput. Chem. 10 (1989), 209-220. https://doi.org/10.1002/jcc.540100208

[23] J. J. P. Stewart, Optimization of parameters for semiempirical methods II. Applications, $J$. Comput. Chem. 10 (1989), 221-264. https://doi.org/10.1002/jcc.540100209

[24] A. R. Leach, Molecular Modeling, Essex, UK: Longman, 1997.

[25] W. Kohn and L. J. Sham, Self-consistent equations including exchange and correlation Effects, Phys. Rev. A 140 (1965), 1133-1138. https://doi.org/10.1103/PhysRev.140.A1133

[26] R. G. Parr and W. Yang, Density Functional Theory of Atoms and Molecules, London: Oxford University Press, 1994.

[27] A. D. Becke, Density-functional exchange-energy approximation with correct asymptotic behavior, Phys. Rev. A 38 (1988), 3098-3100. https://doi.org/10.1103/PhysRevA.38.3098

[28] S. H. Vosko, L. Wilk and M. Nusair, Accurate spin-dependent electron liquid correlation energies for local spin density calculations: a critical analysis, Can. J. Phys. 58 (1980), 1200-1211. https://doi.org/10.1139/p80-159

[29] C. Lee, W. Yang and R. G. Parr, Development of the Colle-Salvetti correlation-energy formula into a functional of the electron density, Phys. Rev. B 37 (1988), 785-789. https://doi.org/10.1103/PhysRevB.37.785

[30] SPARTAN 06, Wavefunction Inc., Irvine CA, USA.

[31] R. O. C. Norman, Principles of Organic Synthesis, London: Methuen, 1970.

[32] P. J. Durant and B. Durant, Introduction to Advanced Inorganic Chemistry, London: Longman, 1972.

[33] Spartan, Molecular Modeling in Physical Chemistry, Irvine CA, USA: Wavefunction Inc., 2005. 